\title{
Atenção visual: estudos comportamentais da seleção baseada no espaço e no objeto
}

\author{
Joaquim Carlos Rossini \\ Universidade Federal de Uberlândia \\ César Galera \\ Universidade de São Paulo - Ribeirão Preto
}

\begin{abstract}
Resumo
Apesar de intensamente pesquisados nos últimos trinta anos, os processos cognitivos relacionados à atenção visual humana ainda apresentam várias lacunas e instigam investigações sobre os mecanismos de seleção e integração da informação relevante contida no ambiente. Basicamente, os esforços para a compreensão desta arquitetura cognitiva estão centrados em dois grandes modelos teóricos sobre a atenção visual: um baseado na localização espacial ocupada pelos objetos no campo visual e outro baseado nas características do objeto a ser atendido. A revisão realizada neste artigo busca sistematizar algumas contribuições experimentais importantes a respeito desses modelos bem como evidenciar algumas particularidades da natureza dos processos envolvidos na mobilização da atenção visual humana.
\end{abstract}

Palavras-chave: atenção visual; modelos da atenção visual; psicologia cognitiva

\begin{abstract}
Visual attention: behavioral studies about location-based and object-based selection. The study of visual attention has become an important topic in cognitive psychology research in the last 30 years. However, many questions about the nature of attention are not well understood. The efforts to understand this cognitive architecture are centered in two great theoretical models on the visual attention: the first model supports that visual attention is allocated on visual space (location-based visual attention) while a second model supports that attention could select the objects representations per se (object-based visual attention). In this review, we pooled important researches about these two seminal models and some questions concerning the human visual attention were examined.
\end{abstract}

Keywords: visual attention; visual attentional models; cognitive psychology

$\mathrm{N}$ este exato momento você está iniciando uma tarefa de leitura. Esta tarefa, aparentemente simples, exige que o seu sistema cognitivo selecione a informação mais relevante para a compreensão deste texto. Provavelmente muitos outros estímulos visuais, auditivos e tácteis estão presentes simultaneamente no ambiente ao seu redor, e devem ser suprimidos ou atenuados para que você consiga se “concentrar” na tarefa de leitura. Este fenômeno mental é conhecido como atenção seletiva.

A atenção seletiva é a capacidade mental de selecionar apenas uma pequena parcela da informação contida no ambiente em detrimento da grande quantidade de estímulos disponíveis ao nosso redor. Talvez o primeiro pesquisador a investigar de maneira sistemática este fenômeno tenha sido Herman von Helmholtz (Wright \& Ward, 1998). Ele conduziu um experimento considerado por muitos como a primeira demonstração científica da capacidade do sistema visual huma- no em direcionar a atenção para uma determinada área do campo visual, na ausência de movimentos oculares. Para demonstrar esta capacidade, Helmholtz fixou o seu olhar em um pequeno ponto iluminado no centro de um campo com letras impressas não iluminadas. A seguir, este campo visual era iluminado rapidamente pelo clarão de uma faísca elétrica. Esta iluminação era tão breve que não permitia nenhum movimento ocular durante a apresentação dos estímulos. Nesta condição, ele foi incapaz de distinguir as letras ao redor do ponto de fixação. No entanto, se antecipadamente ele direcionava a sua "atenção" para uma parte específica do campo visual, o reconhecimento das letras ali representadas tornava-se possível. Helmholtz interpretou este resultado como um reflexo da capacidade de direcionamento interno dos recursos atentivos para uma área específica do campo visual. Muitos pesquisadores, após este experimento pioneiro, levantaram questões importantes acerca da atenção seletiva, entre eles, 
William James (1890) e Edward B. Titchener (1908), de acordo com Pashler (1998). Mas foi apenas a partir da década de 1950, com o advento da psicologia cognitiva, que a investigação sobre atenção seletiva foi vigorosamente retomada, graças, em grande parte, aos trabalhos seminais realizados por eminentes pesquisadores, como Colin Cherry (1953), Donald Broadbent (1958) e Ulric Neisser (1967).

Uma importante contribuição neste período foi apresentada por Broadbent (1958) que formulou uma influente teoria sobre os processos atentivos e que norteou boa parte das investigações na área durante a segunda metade do século XX. Segundo esta teoria, conhecida como teoria do filtro atentivo, o processamento da informação está sujeito a, pelo menos, dois estágios. Durante o primeiro estágio os estímulos são detectados e selecionados conforme suas características físicas. Neste processo, a atenção atua como um filtro que permite que apenas os estímulos com características físicas relevantes sejam processados em estágios posteriores. Nesta concepção os estímulos não relevantes seriam simplesmente bloqueados e desconsiderados pelo sistema cognitivo (para uma revisão, veja Lachter, Forster, \& Ruthruff, 2004).

Ainda na década de 1960, surgiram algumas inconsistências práticas na idéia de um "gargalo atentivo" que atua bloqueando estímulos não relevantes. Nessa época, a maioria dos estudos sobre a atenção seletiva priorizava a utilização de estímulos auditivos, sobretudo nas pesquisas envolvendo a técnica da escuta dicótica, ou que investigavam o paradigma conhecido como o problema do coquetel. Neste tipo de tarefa o participante deve seguir uma mensagem apresentada a um dos ouvidos enquanto ignora uma outra mensagem apresentada simultaneamente ao outro ouvido. Assim, em um primeiro estágio do processamento da informação atribuído à detecção das características físicas do estímulo, tanto a informação apresentada ao ouvido direito quanto ao ouvido esquerdo seriam processadas. Todavia, de acordo com a teoria do filtro atentivo, apenas a informação apresentada ao ouvido atendido deveria ser processada em níveis cognitivos mais complexos. No entanto, vários resultados experimentais mostraram que a seletividade deste suposto filtro atentivo pode ser parcial, e que em certas circunstâncias a informação, a priori não atendida, pode ser processada em níveis cognitivos superiores (Treisman, 1960, 1964a, 1964b). Esse fato levou à elaboração de uma teoria alternativa àquela proposta inicialmente por Broadbent, conhecida como teoria da atenuação do sinal. Segundo esta teoria, a atenção seria responsável por uma atenuação dos estímulos não relevantes, e atuaria como um mecanismo enfraquecedor do sinal irrelevante para o sistema. Para estímulos particularmente potentes, no entanto, os efeitos da atenuação atentiva não seriam suficientes para impedir o seu processamento em estruturas cognitivas superiores. Tanto o modelo proposto por Broadbent quanto o modelo da atenuação do sinal ficaram conhecidos como modelos de seleção precoce (early selection model). Um modelo alternativo ao enfoque de descarte precoce foi proposto por Deutsch e Deutsch (1963) e ficou co- nhecido como modelo de seleção tardia (late selection model). Nesta suposição, a informação é processada ou descartada através de um processamento cognitivo sucessivo, não apenas baseado nas características físicas do objeto, mas também na sua representação mental.

A partir da década de 1970, houve certa mudança no enfoque das pesquisas nesta área e um maior interesse foi atribuído aos estudos que utilizavam estímulos visuais. Esta tendência visual no estudo da atenção perdura até os dias atuais e tem permitido a elaboração de vários modelos sofisticados a respeito do processamento atentivo da informação, embora essencialmente fundamentado no funcionamento do sistema visual. Alguns desses modelos incluem a distinção entre processos automáticos e processos controlados proposta por Schneider e Shiffrin (1977), ou a necessidade de uma integração atentiva da informação multidimensional proposta na teoria da integração das características, elaborada por Treisman e Gelade (1980) e Treisman e Sato (1990). Outra contribuição expressiva neste campo foi apresentada nas pesquisas seminais realizadas por Posner, Snyder e Davidson (1980), sobre as características da atenção visual baseada nas localizações espaciais dos estímulos e da capacidade do sistema atentivo em direcionar os seus recursos para uma parte específica do campo visual (Cave \& Bichot, 1999).

Nos últimos quinze anos, a atenção visual tem sido estudada através de dois modelos gerais: um baseado na localização dos estímulos no espaço e outro baseado nas características intrínsecas do objeto, independente da sua posição espacial. Estes dois modelos guardam uma relação estreita com duas categorias de operações distintas. A primeira apresenta um processamento basicamente balizado por parâmetros fisiológicos da detecção do estímulo (e.g., aspectos espaciais e temporais). Estes processos são freqüentemente definidos como processos de baixa ordem, ou processos bottom-up. A segunda categoria de operações apresenta processos centrados nas diretrizes internas dos sujeitos, sendo balizada por parâmetros e metas estabelecidas pelo sistema cognitivo sob influência direta dos processos mnemônicos e das representações mentais. Estas operações são definidas como processos de alta ordem, ou processos top-down (Pashler, Johnston, \& Ruthruff, 2001). Em condições normais, tanto processos de baixa ordem quanto processos de alta ordem atuam na seleção dos estímulos mais relevantes no campo de busca. Nesta condição, a atenção visual pode ser considerada como um processo intermediário que integra coerentemente os objetos.

Na concepção da atenção seletiva baseada no espaço os estímulos são selecionados a partir de uma representação perceptiva interna dos objetos. Esta é a idéia fundamental de toda uma série de modelos cognitivos para o processamento da informação visual conhecidos como modelos da atenção visual baseada na localização espacial (location-based visual attention), nos quais a atenção seleciona os estímulos a partir de uma representação espacial primária em que o objeto é representado por dimensões simples não integradas. Nesta circunstância, a focalização dos 
recursos atentivos é responsável pela integração das características do objeto. Assim, o objeto composto por várias dimensões é selecionado e posteriormente processado em operações cognitivas de alta ordem.

Uma metáfora muito utilizada, sobretudo após os trabalhos realizados durante a década de 1980, tem sido a comparação da atenção visual a um holofote que se desloca através de uma superfície não iluminada. Este modelo é conhecido como modelo do holofote atentivo, ou (attention spotlight model). Neste modelo a atenção visual é comparada a um feixe de luz sobre uma superfície não iluminada. A atenção, neste modelo, move-se através de um campo visual interno de representações e seleciona e integra os estímulos com base nas suas localizações. Nesta situação o foco atentivo apresenta invariavelmente uma forma circular constante.

Para demonstrar esta característica espacial na distribuição dos recursos da atenção visual Posner et al. (1980) delinearam uma tarefa experimental em que os participantes eram solicitados a fixar o olhar em um ponto central no campo visual, preparando-se para responder a um estímulo luminoso simples. A seguir, uma possível posição à direita ou à esquerda do ponto de fixação era indicada de maneira simbólica (e.g., uma seta sobre o ponto de fixação apontando o lado em que o estímulo provavelmente ocorreria) ou de modo direto (e.g., a posição em que o estímulo provavelmente ocorreria era sinalizada diretamente através do aparecimento súbito e breve de um ponto luminoso). Logo após a apresentação dessa “dica”, o alvo (que poderia ser outro ponto luminoso) era apresentado, e os participantes realizavam uma tarefa de reação simples, acusando a detecção do alvo ao pressionar um botão. Invariavelmente os participantes respondiam mais rapidamente quando o alvo era apresentado na posição indicada pela dica visual válida do que quando era apresentado na posição oposta da indicada pela dica visual (dica inválida). Este custo temporal associado a uma dica inválida foi interpretado como uma evidência do deslocamento espacial analógico do foco da atenção.

Essa distribuição espacial da atenção recebeu um apoio robusto de vários resultados experimentais obtidos ao longo das últimas décadas. Por exemplo, Eriksen e Eriksen (1974) sugeriram a utilização de um paradigma de competição pela resposta para demonstrar esta natureza espacial da atenção. Neste paradigma, também conhecido como tarefa flanker, os participantes eram solicitados a identificar a letra central de um arranjo horizontal de letras. A letra-alvo era ladeada por letras intrusas, flankers, irrelevantes à tarefa de identificação da letra-alvo. Estas letras intrusas eram compatíveis com a letra-alvo (e.g., "A A A”, sendo a letra-alvo sempre a letra central do arranjo) ou incompatível com a letra-alvo (e.g., "A O A”). Invariavelmente, o tempo de reação era maior quando as letras intrusas eram incompatíveis com o alvo, do que quando eram compatíveis. Este resultado foi interpretado como o reflexo de um processo de competição pela resposta baseado no espaço. Outro resultado importante observado neste tipo de experimento foi a diminuição da interferência causada pela letra intrusa com o aumento da distância entre ela e o alvo. Este fato também evidenciou uma característica espacial na distribuição dos recursos atentivos no campo visual (Müller, Mollenhauer, Rösler, \& Kleinschmidt, 2005; Sanders \& Lamers, 2002).

Outro aspecto bastante investigado neste enfoque baseado na localização espacial dos estímulos são os processos atentivos que possibilitam a focalização dos recursos sobre uma determinada área do campo visual (Eriksen \& Hoffman, 1972; Eriksen \& St. James, 1986; Eriksen \& Yeh, 1985; LaBerge, 1983; Muller et al., 2005).

LaBerge (1983) investigou a questão do tamanho do foco atentivo através de duas tarefas sucessivas. A primeira tarefa foi dividida em dois tipos. No primeiro tipo, o participante era solicitado a determinar se uma linha composta por cinco letras, brevemente apresentada, formava um nome próprio ou não. Esta tarefa foi denominada tarefa-palavra (word task). Em um segundo tipo de tarefa primária, o participante era solicitado a identificar se a letra central do arranjo pertencia ao conjunto de letras de $A$ a $G$. Esta tarefa foi denominada tarefa-letra (letter task). Imediatamente após esta primeira tarefa (Experimento 1), ou depois de um intervalo de $500 \mathrm{~ms}$ (Experimento 2), um estímulo-alvo (o número 7) era apresentado em uma das cinco posições ocupadas anteriormente pelas letras. Os participantes eram instruídos a pressionar um botão, o mais rápido possível, para indicar a detecção do alvo.

Nesta situação, a primeira tarefa (palavra ou letra) foi delineada para provocar uma distribuição específica da atenção, enquanto que a segunda tarefa foi usada para mapear essa distribuição no espaço. LaBerge estabeleceu a hipótese de que a tarefa-palavra poderia provocar uma distribuição uniforme da atenção por todas as cinco posições ocupadas pelas letras do arranjo. Por outro lado, a tarefa-letra permitiria apenas uma distribuição da atenção focalizada sobre a posição central das cinco posições possíveis no arranjo. A sua hipótese era de que a distribuição da atenção causada pela tarefa primária poderia ser expressa na redução do tempo necessário para a detecção do estímulo-alvo. Essa hipótese foi confirmada da seguinte maneira: o tempo de reação para a detecção do estímulo-alvo após a tarefa-palavra foi o mesmo, independentemente da posição em que o estímulo-alvo foi apresentado. No entanto, após a tarefa-letra, a detecção do estímulo-alvo foi mais rápida quando este foi apresentado na posição central do arranjo, o que produziu uma curva de tempo de resposta por posição espacial com a forma de um $V$. O resultado desse experimento sugeriu, portanto, que o tamanho do foco atentivo apresentava a capacidade de adaptarse à demanda da tarefa.

Em estudos posteriores, Eriksen e Yeh (1985) e Eriksen e St. James (1986) utilizaram uma tarefa de discriminação para investigar a capacidade do foco atentivo em ajustar-se ao tamanho de uma área determinada pela demanda da tarefa. Estes autores propuseram que o foco atentivo poderia ser metaforicamente melhor comparado ao zoom de uma lente de aumento do que ao foco constante de um holofote, como sugerido pelo modelo spotlight. Para demonstrar esta característica, Eriksen e St. James (1986) elaboraram uma tarefa de 
discriminação em que os participantes eram solicitados a identificar a letra $S$ ou $C$ o mais rápido possível após a apresentação de uma, duas ou três indicações (dicas) da possível localização do alvo. A letra-alvo era apresentada com outras sete letras distratoras, que podiam ser neutras $(N, H$, ou $A)$ ou "competidoras" compatíveis ou incompatíveis com a letraalvo ( $S$ ou $C$ ). As letras foram apresentadas em oito posições dispostas em um arranjo circular com o diâmetro de $1,5^{\circ}$ de ângulo visual e a letra-alvo era sempre apresentada em uma das posições indicadas. Quatro variáveis foram manipuladas: (a) o intervalo entre a apresentação das dicas e dos estímulos imperativos (stimulus onset asynchrony - SOA); (b) o número de posições pré-indicadas (1, 2, ou 3); (c) a presença ou não de uma letra competidora compatível ou incompatível com a letra-alvo, apresentada fora das posições pré-indicadas (similar à tarefa flanker utilizada por Eriksen \& Eriksen, 1974), e (d) a distância entre as letras competidoras e as posições pré-indicadas. Os resultados mostraram: (a) um efeito significativo do intervalo entre a apresentação da(s) dica(s) e do alvo (quanto maior era o intervalo entre dica e alvo, menor era o efeito das letras competidoras incompatíveis no aumento no tempo de reação); (b) um efeito significativo no tempo de reação vinculado ao número de posições pré-indicadas (quanto maior o número de posições pré-indicadas, maior era o tempo de reação dos participantes); e (c) as letras competidoras apresentaram o efeito esperado no tempo de reação (as letras incompatíveis causaram um aumento significativo no tempo de reação). Os autores concluíram que o tamanho do foco atentivo pode ser manipulado através de dicas preditivas, e que há uma relação inversa entre o tamanho do foco atentivo e a eficiência para o processamento da informação apresentada dentro da área focalizada. Assim, o processo de focalização da atenção visual teria características muito semelhantes, não às de um holofote, mas sim ao funcionamento de uma poderosa lente de aumento. Este modelo ficou conhecido como modelo da lente zoom, ou zoom lens model.

A questão do ajuste do tamanho do foco atentivo de maneira semelhante ao funcionamento de uma lente zoom foi estudada por Castiello e Umiltà (1990). Esses autores adotaram um paradigma semelhante ao utilizado por Posner et al. (1980), no qual duas regiões do campo visual eram igualmente delimitadas. Nos experimentos realizados por Castiello e Umiltà essas regiões apresentavam áreas distintas de prova para prova. Estas áreas eram definidas por molduras quadrangulares, ou dicas espaciais, com dimensões de $1 \mathrm{x} 1,2 \mathrm{x} 2$ ou 3x3 graus de ângulo visual, apresentadas a 10 graus à direita e à esquerda de um ponto de fixação central. As dicas permaneciam visíveis até o final da prova, e após um intervalo de 40 ou 500 ms o alvo era apresentado no interior de uma delas.

O Experimento 1A de Castiello e Umiltà (1990) apresentava provas neutras e válidas. Nas provas neutras, os dois quadrados eram apresentados simultaneamente. Nas provas válidas, apenas um dos quadrados era apresentado, indicando sempre a posição do alvo. No Experimento 1B, provas válidas e inválidas foram utilizadas. Para tanto, dois quadra- dos foram apresentados simultaneamente e eram compostos por dois tipos de margens: uma formada por linhas contínuas (dica válida) e outra formada por linhas tracejadas (dica inválida). Os participantes eram instruídos a direcionar a atenção apenas para a dica composta por margens contínuas. Em $80 \%$ das provas o alvo era apresentado dentro da região válida (provas válidas) enquanto que nos $20 \%$ de provas restantes, o alvo era apresentado dentro da área delimitada pela dica tracejada (provas inválidas). No Experimento 2, apenas dois tamanhos de dicas foram utilizados (1 ou 3 graus de ângulo visual) em um delineamento semelhante ao utilizado no Experimento 1A, com apresentações de dicas válidas e neutras. De maneira distinta dos dois primeiros experimentos, o alvo foi apresentado sempre após um período de exposição da dica igual a $500 \mathrm{~ms}$. Outra diferença importante era que o alvo podia ser apresentado em cinco posições dentro da dica com 3 graus de lado, ou em quatro posições externas e uma interna quando a dica apresentava apenas 1 grau de lado. De maneira geral os resultados obtidos nos Experimentos 1A e 1B mostraram: (a) que a eficiência dos participantes melhorou quando o tempo de apresentação da dica foi de 500 ms; e (b) que há uma diferença significativa no processamento da informação inversamente relacionada ao tamanho da área focalizada.

Posteriormente, Maringelli e Umiltà (1998) investigaram a possibilidade dos processos de orientação e de focalização dos recursos atentivos ocorrerem de maneira paralela, competindo assim, pelos mesmos recursos. Para evitar esta possível sobreposição de processos os autores eliminaram a etapa de orientação da atenção apresentando a dica espacial e o estímulo-alvo sempre na mesma posição no centro da tela de apresentação. Os resultados gerais desta investigação mostraram que na ausência do processo de orientação a focalização dos recursos atentivos pode ser iniciada em momentos precoces do processamento da informação visual. Esses autores sugerem que a focalização dos recursos atentivos pode ser compreendida como uma reação automática, ou reflexa, atribuída ao aparecimento de um novo objeto no campo visual.

Esta dinâmica temporal do processo de focalização atentiva foi investigada de maneira mais sistemática por Benso, Turatto, Mascetti e Umiltà (1998), que procuraram determinar o tempo necessário para a focalização dos recursos, e por quanto tempo estes recursos podem ser mantidos focalizados. Esses autores utilizaram um paradigma experimental que envolvia uma tarefa de reação simples a um estímulo apresentado dentro de uma dica espacial circular com 2,5 ou 7,5 graus de ângulo visual. Um pequeno ponto verde era brevemente apresentado antes da dica espacial em uma posição congruente à posição efetiva da dica no campo visual. Este sinal foi utilizado com o intuito de permitir que o processo de orientação da atenção fosse finalizado antes do processo de focalização ser iniciado. A manipulação da variável SOA mostrou que o processo de focalização ocorre entre 33 e 66 ms, e pode ser mantido por um intervalo de aproximadamente 500 ms. Além desse intervalo, o foco não pode 
ser mais mantido e o sistema atentivo volta a um estado difuso. Estes resultados também foram obtidos quando a dica e o estímulo-alvo foram apresentados no centro da tela, eliminando assim uma possível influência dos movimentos oculares. Os autores concluíram que o processo de distribuição dos recursos atentivos no campo visual envolve, pelo menos, três processos distintos: orientação, focalização e manutenção ativa do foco.

Turatto, Benso, Facoetti, Galfano, Mascetti e Umiltà (2000) obtiveram resultados que acrescentaram evidências acerca da característica automática da focalização atentiva. Estes autores utilizaram um paradigma semelhante ao utilizado por Benso et al. (1998). Em linhas gerais, esses autores obtiveram resultados que sugerem que o tamanho do foco atentivo pode se ajustar automaticamente ao tamanho de um novo objeto apresentado no campo visual. Os autores sugerem, então, que o processo de focalização é independente do processo de orientação atentiva, e que este mecanismo automático de ajuste é baseado no aparecimento abrupto de um novo objeto dentro da área já atendida pelo foco da atenção.

Em suma, todos estes modelos apresentam uma base comum: a atenção visual seleciona a informação relevante proveniente de representações internas fundamentadas nas localizações dos estímulos visuais. Esta seleção ocorre sob o foco dos recursos atentivos que podem ser comparados metaforicamente ao "feixe de luz de um holofote que se desloca sobre uma superfície escura”. Este foco tem características mais estáveis no modelo spotlight, proposto por Posner et al. (1980), e características mais flexíveis, quanto ao seu tamanho, no modelo zoom lens, proposto por Eriksen e Yeh (1985) e Eriksen e St. James (1986). Tanto o modelo spotlight quanto o modelo zoom lens, sugerem uma forma circular ou oval para o foco atentivo, enquanto que apenas o modelo zoom lens prediz uma relação inversa entre o tamanho do foco atentivo e a eficiência no processamento da informação contida dentro do foco da atenção.

Além da flexibilidade relacionada ao seu tamanho, alguns autores têm considerado a possibilidade do foco atentivo apresentar uma flexibilidade relacionada também a sua forma. Por exemplo, Egly e Homa (1984) apresentaram a sugestão de que o foco da atenção poderia ser mobilizado sobre regiões concêntricas localizadas a diferentes distâncias de um ponto de fixação central. Os participantes deste estudo foram solicitados a identificar (Experimento 1) ou localizar (Experimentos 2 e 3) uma letra apresentada dentro de um dos três anéis concêntricos, e receberam instruções explícitas para focar a atenção na região anelar na qual o alvo provavelmente seria apresentado. Os resultados destes experimentos mostraram que a focalização da atenção em um dos anéis tornava mais eficiente o processamento dos estímulos apresentados na região indicada. Assim, os autores sugeriram que a atenção pode assumir a forma de um anel (ring model), ou pelo menos ser distribuída de uma maneira não contínua pelo espaço de representações visuais. Juola, Bouwhuis, Cooper e Warner (1991) replicaram este estudo utilizando uma tarefa de tempo de reação de escolha e obtiveram resultados se- melhantes que corroboram a idéia de que a atenção pode se ajustar a formas particulares. Mais recentemente, Müller e Hübner (2002) obtiveram resultados neurofisiológicos que também apóiam a idéia que o foco da atenção visual pode assumir formas anelares.

Em uma série de cinco experimentos, Usai, Umiltà, e Nicoletti (1995) investigaram a possibilidade de um controle voluntário da forma do foco atentivo. Os participantes desse estudo foram solicitados a fixar o olhar no centro da tela e pressionar o mais rápido possível uma tecla assim que o alvo fosse apresentado. O alvo poderia ser apresentado dentro ou fora de uma área delimitada por um polígono simples (hexágono ou losango). Os participantes também foram instruídos a ignorar voluntariamente uma área específica dentro das figuras, denominada pelos autores de área crítica. O resultado deste estudo sugeriu que os participantes foram capazes de mobilizar a atenção sobre a área demarcada pelas figuras, mas foram incapazes de ignorar voluntariamente a informação apresentada dentro da área crítica. De acordo com os autores, este resultado fortalece o modelo de lente zoom, e sugere que o foco atentivo apresenta uma forma unitária que pode se expandir para abranger múltiplos estímulos. Todavia, esta expansão não está sob um controle voluntário e não apresenta uma forma flexível o suficiente para excluir ou ignorar áreas específicas da região atendida.

Se o foco atencional não tem flexibilidade suficiente para ignorar estímulos apresentados em áreas específicas, existe certa flexibilidade para focalizar regiões com orientações específicas. Por exemplo, Panagopoulus, von Grünau, e Galera (2004), investigaram uma situação de busca visual em que o participante era informado quando o alvo e os distratores seriam apresentados em uma região vertical ou horizontal no centro da tela. Esta pré-indicação da região a ser atendida resultou em uma maior eficiência na discriminação do alvo quando os estímulos, de fato, eram apresentados na região indicada. As dicas válidas, que ocorreram em 75\% das provas, supostamente permitiram que o sistema atentivo utilizasse, de maneira voluntária, a forma da dica para melhorar o desempenho na tarefa de busca visual. Em outro trabalho, Galera, von Grünau, e Panagopoulus (2005) investigaram a possibilidade de um ajuste automático do foco atentivo quanto ao tamanho e a orientação espacial dos recursos no campo visual utilizando dicas exógenas. Os autores utilizaram uma tarefa de busca visual com três distratores e um alvo apresentados em quatro posições ao redor de um ponto de fixação. Este arranjo de estímulos era sempre apresentado no centro do monitor, sozinhos ou dentro de uma forma geométrica. Esta forma geométrica era um quadrado $\left(3,4^{\circ} \times 3,4^{\circ}\right)$ ou um retângulo $\left(9,8^{\circ} \times 3,4^{\circ}\right)$ apresentado com uma orientação vertical ou horizontal no campo visual. Antes da apresentação dos estímulos de busca emoldurados pela figura geométrica, uma dica da forma desta figura era apresentada rapidamente. A dica era válida quando apresentava o mesmo tamanho e orientação da moldura que circundava os estímulos da busca, ou inválida quando apresentava um tamanho ou uma orientação não compatível. De acordo com o critério da 
intencionalidade, a captura automática da atenção pela forma geométrica seria evidenciada por uma facilitação da tarefa de busca nas provas com dicas válidas em relação às provas com dicas inválidas. Os resultados deste estudo mostraram evidencias de que o ajuste do foco atentivo pode ocorrer de maneira automática, e que pode haver uma assimetria na distribuição dos recursos atentivos associada aos meridianos, verticais e horizontais, do campo visual.

Todos estes resultados apontam um importante componente espacial na mobilização dos recursos atentivos, mas não explicam satisfatoriamente vários outros dados referentes à influência das características do objeto nesta mobilização. Vários autores têm investigado a possibilidade, por exemplo, dos recursos atentivos selecionarem os objetos sem a necessidade do processamento da sua posição espacial (Duncan, 1984; Egly, Driver, \& Rafal, 1994; Jordan \& Tipper, 1998; Marshall \& Halligan, 1993; Moore, Yantis, \& Vaughan, 1998; Tipper \& Behrmann, 1996; Tipper \& Weaver, 1998; Treisman, Kahneman, \& Burkell, 1983). Este modelo é conhecido como modelo da atenção baseada no objeto (objectbased visual attention), e sugere que as características do estímulo visual, e não a sua localização espacial, pode ser selecionada e integrada.

Uma demonstração consistente deste modelo foi proposta por Duncan (1984) que apresentou simultaneamente aos participantes dois objetos-alvo sobrepostos, um quadrado e uma linha vertical inclinada. Tanto o quadrado quanto a linha podiam apresentar duas dimensões (características). $\mathrm{O}$ quadrado podia ser grande ou pequeno e apresentar uma abertura do lado direito ou esquerdo. A linha vertical podia ser tracejada ou pontilhada e inclinada para a direita ou para a esquerda. Os participantes foram solicitados a relatar uma ou duas dimensões dos alvos apresentados. Quando duas dimensões eram relatadas, elas poderiam ser do mesmo objeto (e.g., quadrado pequeno, aberto na direita) ou de objetos distintos (e.g., quadrado pequeno, linha tracejada). Os participantes não apresentaram dificuldades em relatar uma ou duas dimensões das quatro dimensões apresentadas. No entanto, eles foram mais precisos em relatar duas dimensões de um mesmo objeto do que relatar duas dimensões em objetos distintos. Estes resultados não foram compatíveis com a teoria da atenção baseada no espaço, já que as dimensões dos objetos eram eqüidistantes. Nesta condição, de acordo com a teoria baseada no espaço, os participantes deveriam apresentar um desempenho igual para o relato de todas as dimensões dos objetos, uma vez que todas as características dos dois objetos ocupavam a mesma posição no espaço, o que não foi observado nos resultados experimentais obtidos por Duncan. O autor interpretou este fato como um indício favorável a uma distribuição dos recursos baseada no objeto a ser selecionado, e não no espaço por ele ocupado.

Uma questão metodológica importante para o estudo do foco atentivo diz respeito à real possibilidade de dissociação entre posição espacial e as demais características do objeto. Em outras palavras, é possível o processamento visual atentivo de um objeto sem a sua referência espacial? Ou ain- da, é possível que um objeto seja atentivamente percebido sem que a sua posição no espaço seja processada?

Estas perguntas podem parecer infundadas frente à crença de que todo objeto ocupa, por definição, um lugar no espaço e, portanto, objeto e espaço são dimensões indissociáveis. No entanto, o cérebro humano parece não obedecer necessariamente esse princípio. Estudos neurológicos com pacientes que apresentavam lesões específicas em certas áreas do cérebro revelaram uma dissociação entre o processamento do objeto e a sua posição no espaço (Robertson, Treisman, Friedman-Hill, \& Grabowecky, 1997; Scholl, 2001). Várias pesquisas sugerem que a atenção pode ser capturada pelas características do objeto, e que os modelos da atenção baseada no espaço e da atenção baseada no objeto, de fato, não se excluem mutuamente (Egly et al., 1994; Kramer \& Jacobson, 1991; Kramer, Weber, \& Watson, 1997; Behrmann, Zemel, \& Mozer, 1998).

Um resultado interessante acerca dos componentes atentivos baseados nas características do objeto e na sua localização espacial foi apresentado por Egly et al. (1994). Esses autores delinearam dois experimentos, um realizado com sujeitos normais e outro com pacientes neurológicos que apresentavam um dano unilateral no córtex parietal direito ou esquerdo. A prova experimental consistia na apresentação simultânea, na tela de um computador, de dois retângulos dispostos lado a lado na horizontal ou na vertical. Após um breve intervalo de exposição, a borda de uma das extremidades de um dos retângulos piscava rapidamente. Imediatamente após esta dica exógena, um pequeno retângulo-alvo era apresentado em três condições: na extremidade préindicada do retângulo (dica válida para localização), na extremidade oposta do retângulo indicado (dica inválida para a localização e válida para o objeto), ou na extremidade do retângulo não indicado (dica inválida tanto para localização quanto para o objeto). A tarefa dos participantes era pressionar, o mais rápido possível, um botão, indicando assim, a detecção do alvo. Os resultados obtidos com os participantes normais mostraram um ganho temporal na detecção tanto para a dica válida para localização espacial quanto para a dica válida somente para o objeto em relação à condição na qual a dica era inválida tanto para localização quanto para o objeto. Isto sugere a ocorrência simultânea de mecanismos atencionais baseados no espaço e no objeto. Os resultados obtidos com os pacientes neurológicos mostraram um padrão de ganho temporal semelhante aos apresentados pelos participantes normais, o que evidenciou uma influência assimétrica dos hemisférios cerebrais tanto na alocação atentiva baseada no espaço quanto no objeto. Os pacientes que apresentavam uma lesão no lobo parietal direito mostraram um déficit relacionado ao componente espacial da atenção enquanto os pacientes que apresentavam uma lesão no lobo parietal esquerdo mostraram um comprometimento no componente atentivo baseado no objeto. Todavia, alguns autores apontaram limitações metodológicas neste experimento e sugeriram explicações alternativas para estes resultados (Stuart, Maruff, \& Currie, 1997; Vecera \& Farah, 1994). Por 
exemplo, Vecera e Farah (1994) sugeriram que a seleção da informação poderia estar intimamente vinculada às características do estímulo e ao tipo de tarefa desempenhada pelo sujeito experimental. Os autores sugeriram que tarefas de detecção simples podem mobilizar recursos de processamento baseados no espaço enquanto que tarefas de discriminação de formas mobilizam recursos baseados no objeto.

Recentemente, Iani, Nicoletti, Rubichi e Umiltà (2001) apresentaram evidências que não apóiam o modelo proposto por Vecera e Farah (1994). Esses autores mostraram que, mesmo em tarefas de detecção simples, as propriedades do objeto são acessadas. Na mesma série de experimentos, Iani e colaboradores investigaram outro aspecto freqüentemente apontado como limitador na metodologia utilizada por Egly et al. (1994). Esta limitação é relacionada a um possível custo temporal vinculado ao deslocamento do foco atentivo ao cruzar as bordas dos retângulos apresentados como objetos na tarefa. Assim, uma explicação alternativa fundamentada apenas no componente espacial da atenção poderia explicar o custo temporal observado na mudança atentiva entre objetos. Nessa situação, o custo temporal observado poderia ser atribuído a uma relativa dificuldade do foco atentivo em transpor as bordas de uma área delimitada por margens bem definidas e não a mudança de objeto. Para investigar esta questão, os autores utilizaram uma versão adaptada do paradigma proposto inicialmente por Egly et al. (1994) que permitiu verificar, em uma mesma tarefa, os aspectos relacionados ao componente da atenção baseado no espaço e no objeto. Os autores apresentaram dois retângulos, semelhantes aos utilizados por Egly e colaboradores, em três condições experimentais. Na primeira condição os achados iniciais de Egly foram replicados. No segundo experimento, os retângulos, apresentados como objetos no campo visual, foram unidos e formaram um objeto único. Os resultados observados mostraram que a união desses dois objetos em uma forma unitária eliminou o custo temporal da reorientação da atenção entre objetos. Este resultado apóia o componente da atenção baseado no objeto, independentemente da tarefa do participante. No terceiro experimento, o mesmo objeto unificado foi utilizado, porém, duas margens foram acrescentadas na área de união dos dois retângulos. Com isto foi possível verificar se as margens entre os objetos eram as responsáveis pelo custo temporal extra no processo de reorientação espacial da atenção. Os resultados mostraram que, quando as margens estão contidas em um único objeto, a sua presença não representa nenhum custo temporal no processo de detecção simples. Tais resultados apóiam uma mobilização atentiva baseada tanto no espaço ocupado pelo objeto quanto nas suas características intrínsecas.

Em conjunto, os resultados dos estudos apresentados acima apóiam a idéia de um sistema de focalização atentiva que apresenta características baseadas no espaço e no objeto. De fato, a idéia que o componente da atenção seletiva baseada no espaço e o componente atentivo baseado no objeto atuam de forma integrada é compartilhada por vários modelos, tanto comportamentais (Soto \& Blanco, 2004), quanto neurofisiológicos (Corbetta, Kincade, Olliger, McAvoy, \& Shuman, 2000; Shipp, 2004). Tal abordagem conciliatória tem proporcionado a idealização de modelos mais flexíveis e dinâmicos acerca da arquitetura cognitiva do processamento da informação.

Contudo, muitas questões permanecem por serem respondidas. Nos últimos 30 anos ocorreu um avanço substancial na nossa compreensão da forma como selecionamos no ambiente as informações relevantes para o nosso comportamento. Os estudos comportamentais que sumariamos neste artigo foram fundamentais para o desenvolvimento não só da psicologia, mas também para outras áreas de investigação, tais como as neurociências cognitivas. Entre as questões importantes e que ainda não foram completamente respondidas, podemos levantar aquela que diz respeito ao papel que o objeto per se tem no processo de mobilização da atenção e, caso tenha algum, como se integra aos processos baseados na alocação espacial destes recursos.

\section{Referências}

Behrmann, M., Zemel, R. S., \& Mozer, M. C. (1998). Object-based attention and occlusion: evidence from normal participants and a computational model. Journal of Experimental Psychology: Human Perception and Performance, 24, 1011-1036.

Benso, F., Turatto, M., Mascetti, G. G., \& Umiltà, C. (1998). The time course of attentional focusing. European Journal of Cognitive Psychology, 10(4), 373-388.

Broadbent, D. (1958). Perception and communication. Londres: Pergamon.

Castiello, U., \& Umiltà, C. (1990). Size of the attentional focus and efficiency of processing. Acta Psychologica, 73, 195-209.

Cave, K. R., \& Bichot, N. P. (1999). Visuospatial attention: Beyond a spotlight model. Psychonomic Bulletin and Review, 6, 204-223.

Cherry, C. (1953). Some experiments on the recognition of speech with one and two ears. Journal of the Acoustical Society of America, 25, 975-979.

Corbetta, M., Kincade, J. M., Ollinger, J. M., McAvoy, M. P., \& Shulman, G. L. (2000). Voluntary orienting is dissociated from target detection in human posterior parietal cortex. Nature: Neuroscience, 3, 292-297.

Deutsch, J. A., \& Deutsch, D. (1963). Attention: some theoretical considerations. Psychological Review, 70, 80-90.

Duncan, J. (1984). Selective attention and the organization of visual information. Journal of Experimental Psychology: General, 113(4), 501-517.

Egly, R., Driver, J., \& Rafal, R. (1994). Shifting visual attention between objects and locations: evidence from normal and parietal lesion participants. Journal of Experimental Psychology: General, 123, 161-177.

Egly, R., \& Homa, D. (1984). Sensitisation in the visual field. Journal of Experimental Psychology: Human Perception and Performance, 10, 778-793.

Eriksen, B., \& Eriksen, C. (1974). Effects of noise letters upon the identification of a target letter in a nonsearch task. Perception \& Psychophysics, 16(1), 143-149.

Eriksen, C., \& Hoffman, J. (1972). Temporal and spatial characteristics of selective encoding from visual displays. Perception \& Psychophysics, 12, 201-204.

Eriksen, C., \& St. James, J. (1986). Visual attention within and around the field of focal attention: a zoom lens model. Perception \& Psychophysics, 40(4), 225-240.

Eriksen, C., \& Yeh, Y. Y. (1985). Allocation of attention in the visual field. Journal of Experimental Psychology: Human Perception and Performance, 11(5), 583-597.

Galera, C., von Grünau, M., \& Panagopoulos, A. (2005). Automatic focusing attention on object size and shape. Psicológica, 26, 147-160. 
Iani, C., Nicoletti, R., Rubichi, S., \& Umiltà, C. (2001). Shifting attention between objects. Cognitive Brain Research, 11, 157-164.

James, W. (1890). The Principles of Psychology (Vol. 1). Nova York: Dover.

Jordan, H., \& Tipper, S. P. (1998). Object-based inhibition of return in static display. Psychonomic Bulletin \& Review, 5, 504-509.

Juola, J. F., Bouwhuis, E. E., Cooper, C., \& Warner, B. (1991). Control of attention around the fovea. Journal of Experimental Psychology: Human Perception \& Performance, 17, 125-141.

Kramer, A. F., \& Jacobson, A. (1991). Perceptual organization and focused attention: the role of objects and proximity in visual processing. Perception \& Psychophysics, 50, 267-284.

Kramer, A. F., Weber, T. A., \& Watson, S. E. (1997). Object based attention selection: grouped arrays or spatially invariant representations. Journal of Experimental Psychology: General, 126, 3-13.

LaBerge, D. (1983). Spatial extent of attention to letters and words. Journal of Experimental Psychology: Human Perception and Performance, 9(3), 371-379.

Lachter, J., Forster, K. I., \& Ruthruff, E. (2004). Forty-five years after Broadbent (1958): still no identification without attention. Psychological Review, 111(4), 880-913.

Maringelli, F., \& Umiltà, C. (1998). The control the attentional focus. European Journal of Cognitive Psychology, 10(3), 225-246.

Marshall, J. C., \& Halligan, P. W. (1993). Visual spatial neglect: a new copying test to assess perceptual parsing. Journal of Neurology, 240, 37-40.

Moore, C. N., Yantis, S., \& Vaughan, B. (1998). Object-based visual selection. Psychological Science, 9, 104-110.

Müller, M. M., \& Hübner, R. (2002). Can the spotlight of attention be shaped like a doughnut? Psychological Science, 13(2), 119-124.

Müller, N. G., Mollenhauer, M., Rösler, A., \& Kleinschmidt, A. (2005). The attentional field has a Mexican hat distribution. Vision Research, 45, 1129-1137.

Neisser, U. (1967). Cognitive Psychology. Nova York: Appleton-Century Crofts.

Panagopoulus, A., von Grünau, M. W., \& Galera, C. (2004). Attentive mechanisms in visual search. Spatial Vision, 17(4-5), 353-371.

Pashler, H. (1998). Introduction. In H. Pashler (Org.), Attention. Hove (Reino Unido): Psychology Press.

Pashler, H., Johnston, J., \& Ruthruff, E. (2001). Attention and performance. Annual Review of Psychology, 52, 629-651.

Posner, M., Snyder, C., \& Davidson, B. (1980). Attention and detection of signals. Journal of Experimental Psychology: General, 109(2), 160-174.

Robertson, L. C., Treisman, A., Friedman-Hill, S. R, \& Grabowecky, M. (1997). The interaction of spatial and object pathways: evidence from Balint's syndrome. Journal of Cognitive Neuroscience, 9, 254-276.
Sanders, A. F., \& Lamers, J. M. (2002). The Eriksen flanker effect revisited. Acta Psychologica, 109, 41-56.

Schneider, W., \& Shiffrin, R. (1977). Controlled and automatic human information processing. Psychological Review, 84, 1-66.

Scholl, B. (2001). Objects and attention: the state of the art. Cognition, 80, 1-46.

Shipp, S. (2004). The brain circuitry of attention. Trends in Cognitive Sciences, 8(5), 223-230.

Soto, D., \& Blanco, M. (2004). Spatial attention and object-based attention: a comparison within a single task. Vision Research, 44, 69-81.

Stuart, G. W., Maruff, P., \& Currie, J. (1997). Object-based visual attention in luminance increment detection? Neuropsychologia, 35, 843-853.

Tipper, S. P., \& Behrmann, M. (1996). Object-centered not scene-based visual neglect. Journal of Experimental Psychology: Human Perception \& Performance, 22, 1261-1278.

Tipper, S. P., \& Weaver, B. (1998). The medium of attention: location-based, object-centered, or scene-based? In R. D. Wright (Org.), Visual Attention (pp. 77-107). Nova York: Oxford University Press.

Titchener, E. B. (1908). Lectures on the elementary psychology of feeling and attention. Nova York. Macmillan.

Treisman, A. (1960). Contextual cues in selective listening. Quarterly Journal of Experimental Psychology, 12, 242-248.

Treisman, A. (1964a). Monitoring and storage of irrelevant messages in selective attention. Journal of Verbal Learning and Verbal Behavior, 3, 449-459.

Treisman, A. (1964b). Selective attention in man. British Medical Bulletin, 20, 12-16.

Treisman, A., \& Gelade, G. (1980). A feature-integration theory of attention. Cognitive Psychology, 12, 97-136.

Treisman, A., Kahneman, D., \& Burkell, J. (1983). Perceptual objects and the cost of filtering. Perception \& Psychophysics, 33, 527-532.

Treisman, A., \& Sato, S. (1990). Conjunction search revisited. Journal of Experimental Psychology: Human Perception \& Performance, 16, 459-478.

Turatto, M., Benso, F., Facoetti, A., Galfano, G., Mascetti, G. G., \& Umiltà, C. (2000). Automatic and voluntary focusing of attention. Perception \& Psychophysics, 62(5), 935-952.

Usai, M. C., Umiltà, C., \& Nicoletti, R. (1995). Limits in controlling the focus of attention. European Journal of Cognitive Psychology, 7(4), 411-439.

Vecera, S., \& Farah, M. (1994). Does visual attention select objects or locations? Journal of_Experimental Psychology, 123(2), 146-160.

Wright, R. D, \& Ward, L. M. (1998). The control of visual attention. In R. D. Wright (Org.). Visual attention (pp. 132-186). Nova York: Oxford University Press.

Joaquim Carlos Rossini, doutor em Psicobiologia pela Universidade de São Paulo (Ribeirão Preto), é professor adjunto no Instituto de Psicologia da Universidade Federal de Uberlândia. Endereço para correspondência: Av. Pará, 1720; Caixa Postal 593; CEP 38401-136. Fone/Fax (34) 3218-2296. E-mail: jrossini@fapsi.ufu.br César Alexis Galera, doutor em Psicologia Experimental pela Universidade de São Paulo, é professor associado na Faculdade de Filosofia, Ciências e Letras de Ribeirão Preto, Universidade de São Paulo. E-mail: cesar_galera@ffclrp.usp.br 